\title{
Examples of four dimensional cusp singularities
}

\author{
By Hiroyasu TsuchiHAshi
}

(Received May 28, 2016)

(Revised Dec. 19, 2016)

\begin{abstract}
We give some examples of four dimensional cusp singularities which are not of Hilbert modular type. We construct them, using quadratic cones and subgroups of reflection groups.
\end{abstract}

\section{Introduction.}

In $[8]$, we showed that an $r$-dimensional cusp singularity $\operatorname{Cusp}(C, \Gamma)$ is obtained from a pair $(C, \Gamma)$ of an open cone $C$ in $\mathbf{R}^{r}$ and a subgroup $\Gamma$ of $G L(r, \mathbf{Z})$ satisfying the following three conditions, where $r$ is an integer greater than 1 .

1. $C$ is strongly convex, i.e., $\overline{x y} \subset C$ for any $x, y \in C$ and $\bar{C} \cap \overline{-C}=\{0\}$.

2. $C$ is $\Gamma$-invariant, i.e., $\gamma C=C$ for all $\gamma \in \Gamma$.

3. $\Gamma$ acts on $D_{C}:=C / \mathbf{R}_{>0}$ properly discontinuously, freely and $D_{C} / \Gamma$ is compact.

$\operatorname{Cusp}(C, \Gamma)$ is obtained by adding a point to the quotient of the tube domain $\mathbf{R}^{r}+$ $\sqrt{-1} C$ under the action of the semidirect product of $\mathbf{Z}^{r}$ and $\Gamma$. In the 2-dimensional case, $\operatorname{Cusp}(C, \Gamma)$ is nothing but a Hilbert modular cusp singularity. Hilbert modular cusp singularities exist in all dimensions greater than 1 , where $C$ is the interior of a simplicial cone and $D_{C} / \Gamma$ is a real torus. It is also known that there exist other higher dimensional cusp singularities of arithmetic type (see [6] and [7, Section 3], for instance). We gave in [8] some 3-dimensional explicit examples of $(C, \Gamma)$ such that $D_{C} / \Gamma$ are not real tori. In 1991, Ishida [3] gave explicit 4-dimensional examples. Until quite recently no other 4-dimensional explicit examples seem to be found. On the other hand, Vinberg [10] gave a way to obtain a subgroup $\Gamma$ of $G L(r, \mathbf{R})$ acting properly discontinuously on a strongly convex open cone $C$ in $\mathbf{R}^{r}$. Here $\Gamma$ is generated by reflections with respect to the hyperplanes containing the $(r-1)$-dimensional faces of a polyhedral cone satisfying certain conditions. Moreover, he gave a simple necessary and sufficient condition for the cone $C$ to be quadratic, i.e., defined by a quadratic polynomial. In this paper, using the results in $[\mathbf{1 0}]$, we give some explicit examples of 4-dimensional pairs $(C, \Gamma)$ such that $\Gamma$ are subgroups of reflection groups.

In Section 1, we show that for any open strongly convex cone $C$ in $\mathbf{R}^{r}$, any subgroup of $G L(r, \mathbf{Z})$ preserving $C$, acts on $D_{C}$ properly discontinuously. In Section 2, we show that if a quadratic polynomial $P$ defines a cone $C$ in $\mathbf{R}^{r}$ and there exists a subgroup $\Gamma$ of $G L(r, \mathbf{Z})$ satisfying the above conditions, then all coefficients of $P$ may be assumed to be

2010 Mathematics Subject Classification. Primary 14J17; Secondary 32S05.

Key Words and Phrases. cusp singularity, quadratic cone, reflection. 
integers and $P(x) \neq 0$ for any point $x$ in $\mathbf{Z}^{r} \backslash\{0\}$. In Section 3, we show that if a quadratic cone $C$ contains a rational polyhedral cone satisfying certain conditions, then there exists a reflection group $\Gamma$ contained in $G L(r, \mathbf{Z})$ and acting on $C$ with compact $D_{C} / \Gamma$. In Section 4, we study the structure of exceptional sets of resolutions of $\operatorname{Cusp}(C, \Gamma)$ for pairs $(C, \Gamma)$ such that $\Gamma$ is a subgroup of a reflection group. Finally, we give three 4dimensional examples of pairs $(C, \Gamma)$ with quadratic $C$, and one with non-quadratic $C$ and a resolution of $\operatorname{Cusp}(C, \Gamma)$ whose exceptional set consists of 4 irreducible components.

\section{Groups acting on cones.}

Let $N$ be a free $\mathbf{Z}$-module of $\operatorname{rank} r>1$, let $M=\operatorname{Hom}(N, \mathbf{Z})$ and let $\langle\rangle:, M \times N \longrightarrow$ $\mathbf{Z}$ be the natural pairing. For an open cone $C$ in $N_{\mathbf{R}}=N \otimes \mathbf{R}$, let $D_{C}=C / \mathbf{R}_{>0}$ and let $p_{C}: C \longrightarrow D_{C}$ be the natural projection.

Definition. $\quad \Gamma_{C}=\{\gamma \in G L(N) \mid \gamma C=C\}$ for an open cone $C$ in $N_{\mathbf{R}}$.

Let $C^{*}=\left\{x \in M_{\mathbf{R}} \mid\langle x, y\rangle>0\right.$ for $\left.y \in \bar{C} \backslash\{0\}\right\}$. If $C$ is an open strongly convex cone in $N_{\mathbf{R}}$, then $\Gamma_{C^{*}}=\left\{{ }^{t} \gamma \mid \gamma \in \Gamma_{C}\right\}$, where ${ }^{t} \gamma$ is the element in $G L(M)$ satisfying $\left\langle{ }^{t} \gamma x, y\right\rangle=\langle x, \gamma y\rangle$ for any elements $x$ and $y$ in $M$ and $N$, respectively.

Theorem 1. If $C$ is an open strongly convex cone in $N_{\mathbf{R}}$, then $\Gamma_{C}$ acts on $D_{C}$ properly discontinuously, i.e., $\{\gamma \in \Gamma \mid \gamma S \cap S \neq \emptyset\}$ is finite for every compact subset $S$ of $D_{C}$.
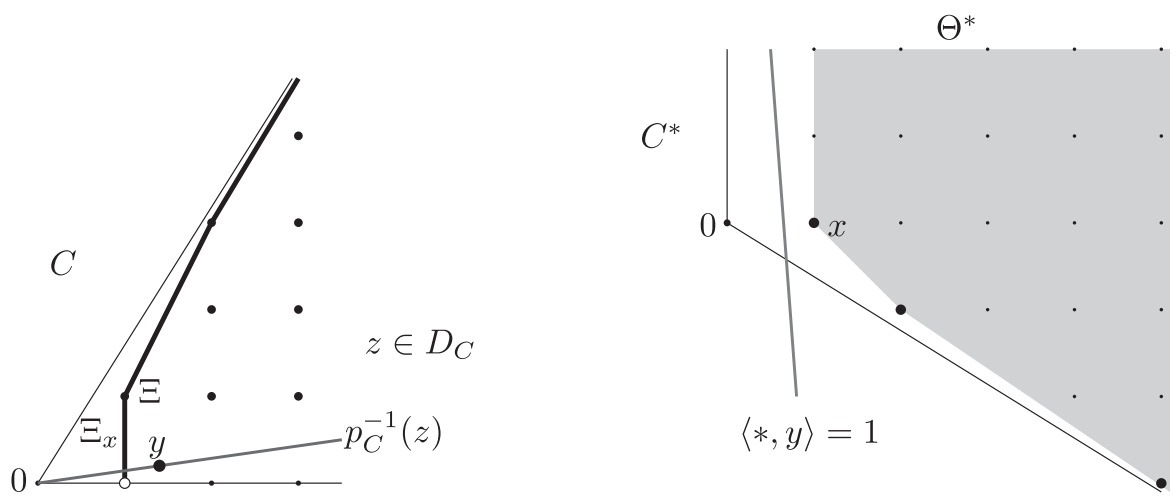

Figure 1.

Proof. Let $\Theta^{*}$ be the convex hull of $C^{*} \cap M$ and let $\Xi$ be the boundary of $\left\{y \in C \mid\langle x, y\rangle \geq 1\right.$ for $\left.x \in \Theta^{*}\right\}$. Then the restriction $p_{C \mid \Xi}: \Xi \longrightarrow D_{C}$ of $p_{C}$ to $\Xi$ is a homeomorphism (see Figure 1). Let $\Xi_{x}=\{y \in \Xi \mid\langle x, y\rangle=1\}$ for each element $x$ in $C^{*} \cap M$. Then $\Xi_{x}$ is closed in $\Xi$. Let $L$ be the set of vertices on $\Theta^{*}$. Then $L$ is contained in $M$ and $\Xi=\bigcup_{x \in L} \Xi_{x}$. For any point $y$ in $\Xi,\left\{x \in L \mid y \in \Xi_{x}\right\} \subset\left\{x \in C^{*} \cap M \mid\langle x, y\rangle=1\right\}$ is finite.

Let $S$ be a compact subset of $D_{C}$. Then $L_{0}=\left\{x \in L \mid S \cap p_{C}\left(\Xi_{x}\right) \neq \emptyset\right\}$ is finite. If $\gamma S \cap S \neq \emptyset$ for an element $\gamma$ in $\Gamma_{C}$, then there exist elements $x_{1}, x_{2}$ in $L_{0}$ with ${ }^{t} \gamma x_{1}=x_{2}$. 
On the other hand, $K=\left\{y \in C \cap N \mid\left\langle x_{1}, y\right\rangle=c\right\}$ contains linearly independent $r$ elements for a positive integer $c$. Then $\left\{\gamma \in \Gamma_{C} \mid{ }^{t} \gamma x_{1}=x_{1}\right\} \subset\left\{\gamma \in \Gamma_{C} \mid \gamma K=K\right\}$ is a finite set. Hence $\left\{\gamma \in \Gamma_{C} \mid{ }^{t} \gamma x_{1}=x_{2}\right\}$ is also finite for any elements $x_{1}, x_{2}$ in $L_{0}$. Therefore, $\left\{\gamma \in \Gamma_{C} \mid \gamma S \cap S \neq \emptyset\right\}$ is finite.

For an open strongly convex cone $C$ with compact $D_{C} / \Gamma_{C}$, there exists a normal subgroup $\Gamma$ of $\Gamma_{C}$ with a finite index acting on $D_{C}$ freely. For example, we obtain such a group as the intersection with the kernel of $S L(N) \rightarrow S L(N / n N)$ for a suitable positive integer $n$.

\section{Quadratic cones.}

We fix a coordinate $\left(X_{1}, X_{2}, \ldots, X_{r}\right)$ of $N$ throughout the rest of this paper. For a homogeneous polynomial $P\left(X_{1}, X_{2}, \ldots, X_{r}\right)$ of $r$ variables, we denote by $C_{P}$ the open cone defined by

$$
\left\{\left(x_{1}, x_{2}, \ldots, x_{r}\right) \in N_{\mathbf{R}} \mid P\left(x_{1}, x_{2}, \ldots, x_{r}\right)>0\right\} .
$$

Definition. We call a cone $C$ in $N_{\mathbf{R}}$ quadratic, if there exists a homogeneous quadratic polynomial $P\left(X_{1}, X_{2}, \ldots, X_{r}\right)$ such that $C$ is a connected component of $C_{P}$.

If a quadratic cone $C$ defined by a polynomial $P$ is strongly convex, then the signature of $P$ is $(1, r-1)$ and $C \cup(-C)=C_{P}$.

THEOREM 2. Let $C$ be a quadratic strongly convex cone in $N_{\mathbf{R}}$ defined by a polynomial $P$. If $D_{C} / \Gamma_{C}$ is compact, then there exists a positive real number $c$ such that all coefficients of $c P$ are integers and $P$ has no isotropic elements in $N$, i.e., $P(x) \neq 0$ for all $x$ in $N \backslash\{0\}$.

Proof. First, we show that there exists a finite set $K$ contained in $C \cap N$ such that the convex hull of $p_{C}\left(\Gamma_{C} K\right)$ is equal to $D_{C}$. Let $\Xi$ be the boundary of the convex hull of $C \cap N$ and let $J=\Xi \cap N$. Then the convex hull of $p_{C}(J)$ is equal to $D_{C}$. On the other hand, $J / \Gamma_{C}$ is finite, because $D_{C} / \Gamma_{C}$ is compact. Hence there exists a finite set $K$ such that $\Gamma_{C} K=J$.

Let $x$ be an element in $K$. We may assume that $P(x)=1$, multiplying $P$ by a positive number. Then $P(\gamma x)=1$ for any element $\gamma$ in $\Gamma_{C}$. Hence all coefficients of $P$ are rational, by the following lemma.

LEMma. There exist $m=r(r+1) / 2$ elements $\gamma_{1}, \gamma_{2}, \ldots, \gamma_{m}$ in $\Gamma_{C}$ and an element $x$ in $K$ such that $f\left(\gamma_{1} x\right), f\left(\gamma_{2} x\right), \ldots, f\left(\gamma_{m} x\right)$ are linearly independent, where $f: N \longrightarrow$ $\mathbf{Z}^{m}$ is the map sending $\left(x_{1}, x_{2}, \ldots, x_{r}\right)$ to $\left(x_{1}^{2}, \ldots, x_{r}^{2}, x_{1} x_{2}, \ldots, x_{r-1} x_{r}\right)$.

Proof. Suppose that $f\left(\gamma_{1} x\right), f\left(\gamma_{2} x\right), \ldots, f\left(\gamma_{m} x\right)$ are linearly dependent for any element $x$ in $K$ and any $m$ elements $\gamma_{1}, \gamma_{2}, \ldots, \gamma_{m}$ in $\Gamma_{C}$. Then $f\left(\Gamma_{C} x\right)$ is contained in an $(m-1)$-dimensional linear subspace of $\mathbf{R}^{m}$. It implies that there exists a homogeneous quadratic polynomial $Q_{x}\left(x_{1}, x_{2}, \ldots, x_{r}\right)$ such that $Q_{x}(\gamma x)=0$ for all $\gamma$ in $\Gamma_{C}$. Since $K$ is finite, there exists a point $x_{0}$ on $\partial C \backslash\{0\}$ such that $Q_{x}\left(x_{0}\right) \neq 0$ for all $x$ in $K$. Then there 
exists a non-zero element $y_{0}$ in $M_{\mathbf{R}}$ such that $\left\langle y_{0}, x_{0}\right\rangle<0$ and that $\left\langle y_{0}, \gamma x\right\rangle>0$ for all $x$ in $K$ and for all $\gamma$ in $\Gamma_{C}$, because there exists a hyperplane $H$ with $H \cap \partial C=\mathbf{R}_{\geq 0} x_{0}$. Hence $D_{C}$ is not equal to the convex hull of $p_{C}\left(\Gamma_{C} K\right)$, a contradiction.

Next, suppose that $P\left(y_{0}\right)=0$ for an element $y_{0}$ in $N \backslash\{0\}$. We may assume that $y_{0}$ is primitive and that $y_{0} \in \partial C$. Let $x_{0}$ be a vertex on the boundary of the convex hull of $\left\{x \in C^{*} \cap M \mid\left\langle x, y_{0}\right\rangle=1\right\}$, which is not empty. Then $x_{0} \in M$ and $y_{0} \in \overline{\Theta_{x_{0}}}$, where

$$
\Theta_{x_{0}}=\left\{y \in C \mid\left\langle x_{0}, y\right\rangle=1,\langle x, y\rangle \geq 1 \text { for } x \in C^{*} \cap M\right\} .
$$

Since $\overline{\Theta_{x_{0}}}$ is compact, $\Theta_{x_{0}} \cap N$ is a finite set. Hence $\Gamma_{0}=\left\{\gamma \in \Gamma_{C} \mid \gamma \Theta_{x_{0}}=\Theta_{x_{0}}\right\}$ is a finite group. Therefore, $p_{C}\left(\Theta_{x_{0}}\right) / \Gamma_{0}$ is not compact. However, $p_{C}\left(\Theta_{x_{0}}\right)$ is closed in $D_{C}$. It implies that $D_{C} / \Gamma_{C}$ is not compact.

In the 2-dimensional case, the converse of the above theorem holds, because $C=$ $\mathbf{R}_{\geq 0} v_{1}+\mathbf{R}_{\geq 0} v_{2}$ for two eigenvectors $v_{1}$ and $v_{2}$ in $N_{\mathbf{R}} \backslash N_{\mathbf{Q}}$ of an element in $S L(N)$.

Proposition 3. An open strongly convex cone $C$ in $N_{\mathbf{R}}$ with compact $D_{C} / \Gamma_{C}$, is quadratic, if and only if there exists a homomorphism $f: N \rightarrow M$ such that $f_{\mathbf{R}}(C)=C^{*}$ and that $f \circ \gamma={ }^{t} \gamma^{-1} \circ f$ for any element $\gamma$ in $\Gamma_{C}$.

Proof. Assume that $C$ is quadratic, i.e., there exists a regular symmetric matrix $A$ of index $(1, r-1)$ such that $C$ is a connected component of $\left\{x \in N_{\mathbf{R}} \mid{ }^{t} x A x>0\right\}$. We may assume that all entries of $A$ are integers, by Theorem 2. Let $f: N \rightarrow M$ be the homomorphism satisfying $\langle f(y), x\rangle={ }^{t} y A x$. Since the index of $A$ is $(1, r-1)$,

$$
\left\{y \in N_{\mathbf{R}} \mid{ }^{t} y A x>0 \text { for } x \in \bar{C} \backslash\{0\}\right\}=C .
$$

Therefore, $f_{\mathbf{R}}(C)=C^{*}$. Let $\gamma$ be any element in $\Gamma_{C}$. Then ${ }^{t} \gamma A \gamma=A$. Hence

$$
\langle f(\gamma y), x\rangle={ }^{t}(\gamma y) A x={ }^{t} y^{t} \gamma A x={ }^{t} y A \gamma^{-1} x=\left\langle f(y), \gamma^{-1} x\right\rangle=\left\langle{ }^{t} \gamma^{-1} f(y), x\right\rangle .
$$

Therefore, $f \circ \gamma={ }^{t} \gamma^{-1} \circ f$.

Conversely, assume that there exists a homomorphism $f: N \rightarrow M$ as in the proposition. We define a symmetric bilinear form on $N_{\mathbf{R}}$ by $x \cdot y=\left\langle f_{\mathbf{R}}(x), y\right\rangle+\left\langle f_{\mathbf{R}}(y), x\right\rangle$. Then there exists a symmetric and integer matrix $A$ with $x \cdot y={ }^{t} x A y$. For any element $\gamma$ in $\Gamma_{C}, \gamma x \cdot \gamma y=x \cdot y$, because $\left\langle f_{\mathbf{R}}(\gamma x), \gamma y\right\rangle=\left\langle{ }^{t} \gamma^{-1} f_{\mathbf{R}}(x), \gamma y\right\rangle=\left\langle f_{\mathbf{R}}(x), y\right\rangle$. Since $f_{\mathbf{R}}(C)=C^{*}, x \cdot y>0$ for any points $x$ and $y$ in $C$. Hence $x \cdot x \geq 0$ for any point $x$ on $\partial C$, because the function $N_{\mathbf{R}} \ni x \mapsto x \cdot x \in \mathbf{R}$ is continuous. Let $\Theta$ be the convex hull of $C \cap N$. Since $\partial \Theta / \Gamma_{C}$ is compact, $\{x \cdot x \mid x \in \partial \Theta\}$ has the maximal value $d$. Let $S_{d}=\left\{x \in N_{\mathbf{R}} \mid x \cdot x=d\right\}$. Then $S_{d} \cap C \subset \Theta$. Since $\Theta$ is closed and $\Theta \cap \partial C=\emptyset$, $S_{d} \cap \partial C=\emptyset$. Hence $x \cdot x=0$ for any point $x$ on $\partial C$. Therefore, $C$ is a connected component of $\left\{x \in N_{\mathbf{R}} \mid x \cdot x>0\right\}$.

The above proposition can be applied to decide whether the cone $C$ is quadratic for a pair $(C, \Gamma)$ satisfying the conditions 1,2 and 3 in Introduction. We give an example. Let $r=3$. Let $S$ be the surface and $\Delta$ be its triangulation obtained from the hexagon in Figure 2, identifying the edges $\overline{v_{1} v_{2}}, \overline{v_{3} v_{4}}$ and $\overline{v_{5} v_{6}}$ with $\overline{v_{2} v_{3}}, \overline{v_{4} v_{5}}$ and $\overline{v_{6} v_{1}}$, respectively. 
Then $\chi(S)=-1$ and the double $\mathbf{Z}$-weight on $\Delta$ as in Figure 2 satisfies the monodromy condition and the convexity condition (see [8, Definitions 1.3 and 1.5]). Hence we obtain a map $\sigma:\{$ all vertices of $\widetilde{\Delta}\} \rightarrow N$ and a homomorphism $\rho: \pi_{1}(S) \rightarrow G L(N)$ such that $\sigma(\gamma v)=\rho(\gamma) \sigma(v)$ for all vertices $v$ of $\widetilde{\Delta}$ and all elements $\gamma$ in $\pi_{1}(S)$ by [8], where $\widetilde{\Delta}$ is the pull-back of $\Delta$ under the universal covering $\varpi: \widetilde{S} \rightarrow S$. Let $C=\mathbf{R}_{>0} \Theta$, where $\Theta$ is the convex hull of the image of $\sigma$, and let $\Gamma=\rho\left(\pi_{1}(S)\right)$. Then the pair $(C, \Gamma)$ satisfies the conditions 1, 2 and 3 in Introduction. There exist vertices $\tilde{v}_{1}, \tilde{v}_{2}, \ldots, \tilde{v}_{6}$ of $\widetilde{\Delta}$ with $\varpi\left(\tilde{v}_{i}\right)=v_{i}$ such that $\overline{\tilde{v}_{1} \tilde{v}_{2} \tilde{v}_{3}}, \overline{\tilde{v}_{3} \tilde{v}_{4} \tilde{v}_{5}}, \overline{\tilde{v}_{5} \tilde{v}_{6} \tilde{v}_{1}}$ and $\overline{\tilde{v}_{1} \tilde{v}_{3} \tilde{v}_{5}}$ are triangles of $\widetilde{\Delta}$. Here we may assume that $\sigma\left(\tilde{v}_{1}\right)=\mathbf{e}_{1}, \sigma\left(\tilde{v}_{3}\right)=\mathbf{e}_{2}$ and $\sigma\left(\tilde{v}_{5}\right)=\mathbf{e}_{3}$, where $\left\{\mathbf{e}_{1}, \mathbf{e}_{2}, \mathbf{e}_{3}\right\}$ is a basis of $N$. Let $\mathbf{f}_{i}=\sigma\left(\tilde{v}_{2 i}\right)\left(=2 \mathbf{e}_{i}+2 \mathbf{e}_{i+1}-\mathbf{e}_{i+2}\right)$ for each $i$ in $\mathbf{Z} / 3 \mathbf{Z}$. Let $\Sigma=\left\{\gamma \tau \mid \gamma \in \Gamma, \tau \prec \mu_{i}, i=\right.$ $0,1,2,3\}$, where $\mu_{0}=\mathbf{R}_{\geq 0} \mathbf{e}_{1}+\mathbf{R}_{\geq 0} \mathbf{e}_{2}+\mathbf{R}_{\geq 0} \mathbf{e}_{3}$ and $\mu_{i}=\mathbf{R}_{\geq 0} \mathbf{e}_{i}+\mathbf{R}_{\geq 0} \mathbf{e}_{i+1}+\mathbf{R}_{\geq 0} \mathbf{f}_{i}$ for $i=1,2,3$. Then $\Sigma$ is a non-singular fan with $|\Sigma| \backslash\{0\}=C$ and $\Gamma$ acts on the set of 1-dimensional cones in $\Sigma$ transitively, because $\Delta$ has only one vertex. Hence we have a resolution of $\operatorname{Cusp}(C, \Gamma)$ whose exceptional set is irreducible.
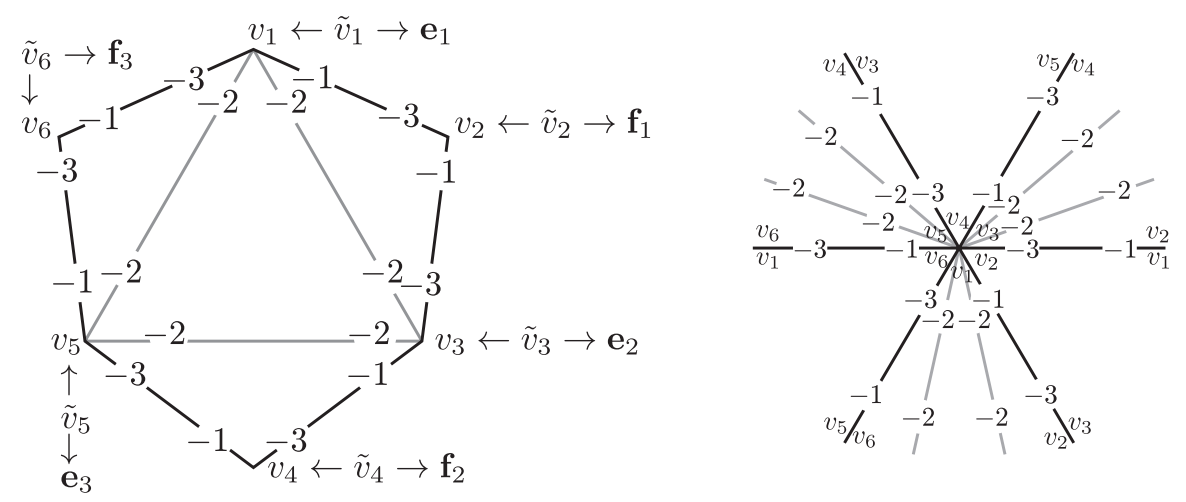

Figure 2.

Proposition 4. The above cone $C$ is not quadratic.

Proof. Let $\gamma_{i}$ be the elements in $G L(N)$ sending $\mathbf{e}_{i}, \mathbf{f}_{i}$ and $\mathbf{e}_{i+1}$ to $\mathbf{f}_{i}, \mathbf{e}_{i+1}$ and $\mathbf{f}_{i}+3 \mathbf{e}_{i+1}-\mathbf{e}_{i}$, respectively for all $i$ in $\mathbf{Z} / 3 \mathbf{Z}$. Then $\gamma_{i}$ are in $\Gamma_{C}$. We easily see that also is in $\Gamma_{C}$ the element sending $\mathbf{e}_{i}$ to $\mathbf{e}_{i+1}$, which we denote by $\delta$. Let $\mathbf{e}_{0}=\mathbf{e}_{1}+\mathbf{e}_{2}+\mathbf{e}_{3}$ and $\mathbf{e}_{0}^{*}=\mathbf{e}_{1}^{*}+\mathbf{e}_{2}^{*}+\mathbf{e}_{3}^{*}$, where $\left\{\mathbf{e}_{1}^{*}, \mathbf{e}_{2}^{*}, \mathbf{e}_{3}^{*}\right\}$ is the basis of $M$ dual to $\left\{\mathbf{e}_{1}, \mathbf{e}_{2}, \mathbf{e}_{3}\right\}$. Then $\delta \mathbf{e}_{0}=\mathbf{e}_{0}$ and ${ }^{t} \delta \mathbf{e}_{0}^{*}=\mathbf{e}_{0}^{*}$. Suppose that there exists an injective homomorphism $f$ : $N \rightarrow M$ satisfying $f \circ \gamma={ }^{t} \gamma^{-1} \circ f$ for any element $\gamma$ in $\Gamma_{C}$. Then $f\left(\mathbf{e}_{0}\right)=c \mathbf{e}_{0}^{*}$ for a non-zero integer $c$, because any fixed point of ${ }^{t} \delta^{-1}$ is on $\mathbf{R e}_{0}^{*}$. We see by an easy calculation that $\gamma_{i} \mathbf{e}_{0}=9 \mathbf{e}_{i}+20 \mathbf{e}_{i+1}-6 \mathbf{e}_{i+2}$ and ${ }^{t} \gamma_{i}^{-1} \mathbf{e}_{0}^{*}=9 \mathbf{e}_{i}^{*}+3 \mathbf{e}_{i+1}^{*}+23 \mathbf{e}_{i+2}^{*}$. Hence $\gamma_{1} \mathbf{e}_{0}+\gamma_{2} \mathbf{e}_{0}+\gamma_{3} \mathbf{e}_{0}=23 \mathbf{e}_{0}$ and ${ }^{t} \gamma_{1}^{-1} \mathbf{e}_{0}^{*}+{ }^{t} \gamma_{2}^{-1} \mathbf{e}_{0}^{*}+{ }^{t} \gamma_{3}^{-1} \mathbf{e}_{0}^{*}=35 \mathbf{e}_{0}^{*}$. It implies $c=0$. Hence $C$ is not quadratic, by Proposition 3 . 


\section{Reflections.}

Let $P$ be a quadratic homogeneous polynomial of $r$ variables with the signature $(1, r-1)$, and let $C$ be a connected component of $C_{P}$. Then $C$ is strongly convex and $C_{P}=C \cup(-C)$. We assume that all coefficients of $P$ are integers with no common divisors greater than 1, throughout this section. Let $B_{P}: N \times N \rightarrow \mathbf{Z}$ be the symmetric bilinear form with $B_{P}(x, x)=2 P(x)$.

Definition. $\quad x \cdot y=B_{P}(x, y)$ for elements $x, y \in N_{\mathbf{R}}$.

We easily see that $\gamma x \cdot \gamma y=x \cdot y$ for any element $\gamma$ in $\Gamma_{C}$. For an element $v$ in $N_{\mathbf{R}}$ with $v \cdot v \neq 0$, we define a linear transformation $\gamma_{v}$ and a hyperplane $H_{v}$ of $N_{\mathbf{R}}$ as follows:

$$
\gamma_{v}: x \mapsto x-2 \frac{x \cdot v}{v \cdot v} v, \quad H_{v}=\left\{x \in N_{\mathbf{R}} \mid x \cdot v=0\right\}
$$

We see by easy calculation that $\gamma_{v}^{2}=\mathrm{id}, \gamma_{v} v=-v, \gamma_{v} x=x$ for any $x$ in $H_{v}$ and $\gamma_{v} x \cdot \gamma_{v} y=x \cdot y$ for any $x, y$ in $N_{\mathbf{R}}$. Hence $\gamma_{v} C=C$ or $-C$. If $v \cdot v<0$, then $\gamma_{v} C=C$, because $C \cap H_{v} \neq \emptyset$. Hence we have:

Proposition 5. If $v$ is an element in $N$ with $v \cdot v<0$ and $2\left(\mathbf{e}_{i} \cdot v / v \cdot v\right) \in \mathbf{Z}$ for each fundamental vector $\mathbf{e}_{i}$, then $\gamma_{v}$ is in $\Gamma_{C}$.

Any element $v$ in $N$ with $v \cdot v=-2$ satisfies the assumption of the above proposition. Let $F_{\gamma}=\{x \in C \mid \gamma x=x\}$ for an element $\gamma$ in $\Gamma_{C}$.

Proposition 6. Let $\gamma$ be an element in $\Gamma_{C}$ with $F_{\gamma} \neq \emptyset$ and $\operatorname{dim} F_{\gamma}=r-1$. Then there exists an element $v$ in $N$ with $\gamma=\gamma_{v}$.

Proof. $\quad r-1$ of the eigenvalues of $\gamma$ are equal to 1 . The other is equal to -1 and $\gamma^{2}=1$, by Theorem 1. Hence there exists a non-zero element $v$ in $N$ with $\gamma v=-v$. For any element $x$ in $N_{\mathbf{R}}$, there exists a real number $c_{x}$ with $x-\gamma x=c_{x} v$, because $\gamma(x-\gamma x)=-(x-\gamma x)$. On the other hand, $\gamma x \cdot v=x \cdot \gamma v$, because $\gamma^{2}=1$. Hence $(x-\gamma x) \cdot v=2 x \cdot v$. Therefore, $c_{x}=2(x \cdot v / v \cdot v)$.

Here we note that an eigenvector $h$ of $\gamma_{v}$ corresponding to the eigenvalue -1 and the linear function $\alpha$ on $N_{\mathbf{R}}$ with $\alpha(h)=2$ and vanishing on $H_{v}$ in [10], are nothing but $v$ and the function $\alpha(x)=2 v \cdot x / v \cdot v$, respectively.

Proposition 7. Let $v$ and $w$ be elements in $N$ with $v \cdot v<0$ and $w \cdot w<0$. If $v \cdot w /(\sqrt{-v \cdot v} \sqrt{-w \cdot w})=0,1 / 2,1 / \sqrt{2}$ or $\sqrt{3} / 2$, then $\left|\gamma_{v} \gamma_{w}\right|=2,3,4$ or 6 , respectively, and $\lambda=\left\{y \in N_{\mathbf{R}} \mid v \cdot y \geq 0, w \cdot y \geq 0\right\}$ is a fundamental domain of the action of $\left\langle\gamma_{v}, \gamma_{w}\right\rangle$ on $N_{\mathbf{R}}$.

Proof. We may assume that $v \cdot v=w \cdot w=-1$ replacing $v$ and $w$ with $v / \sqrt{-v \cdot v}$ and $w / \sqrt{-w \cdot w}$, respectively. Assume that $v \cdot w=\sqrt{3} / 2$. Then $\gamma_{v} \gamma_{w}$ sends $v$ and $w$ to $2 v+\sqrt{3} w$ and $-\sqrt{3} v-w$, respectively. Hence $\left|\gamma_{v} \gamma_{w}\right|=6$. Moreover, 


$$
\lambda=\mathbf{R}_{\geq 0}(-2 v-\sqrt{3} w)+\mathbf{R}_{\geq 0}(-\sqrt{3} v-2 w)+\left\{y \in N_{\mathbf{R}} \mid v \cdot y=w \cdot y=0\right\} .
$$

We see by easy calculation that $r-2 \leq \operatorname{dim}(\gamma \lambda \cap \lambda) \leq r-1$ for any $\gamma$ in $\left\langle\gamma_{v}, \gamma_{w}\right\rangle \backslash\{1\}$. For the other cases, calculation is easier.

If $v \cdot w /(\sqrt{-v \cdot v} \sqrt{-w \cdot w})=-1 / 2,-1 / \sqrt{2}$ or $-\sqrt{3} / 2$, then $\left|\gamma_{v} \gamma_{w}\right|=3$, 4 or 6 , respectively, however, $\operatorname{dim}\left(\gamma_{v} \gamma_{w} \gamma_{v} \lambda \cap \lambda\right)=r$. Let $\sigma$ be an $r$-dimensional rational polyhedral cone. For each $(r-1)$-dimensional face $\tau$ of $\sigma$, we denote by $v(\tau)$ the unique primitive element $v$ in $N$ determined by the condition that $v \cdot y=0$ for all points $y$ in $\tau$ and $v \cdot y \geq 0$ for all points $y$ in $\sigma$.

THEOREM 8. If there exists an $r$-dimensional rational polyhedral cone $\sigma$ satisfying the following three conditions, then $p_{C}(\sigma \backslash\{0\})$ is a fundamental domain of the action of $\Gamma$ on $D_{C}, \Sigma=\{\gamma \lambda \mid \gamma \in \Gamma, \lambda \prec \sigma\}$ is a fan and $|\Sigma|=C \cup\{0\}$, where $\Gamma=\left\langle\gamma_{v(\tau)}\right| \tau \prec$ $\sigma, \operatorname{dim} \tau=n-1\rangle$.

1. $\sigma \backslash\{0\} \subset C$.

2. $v(\tau) \cdot v(\tau)<0$ and $\gamma_{v(\tau)} \in \Gamma_{C}$ for any $(r-1)$-dimensional face $\tau$ of $\sigma$.

3. $v(\tau) \cdot v(\mu) /(\sqrt{-v(\tau) \cdot v(\tau)} \sqrt{-v(\mu) \cdot v(\mu)})=0,1 / 2, \quad 1 / \sqrt{2}$ or $\sqrt{3} / 2$ for any $(r-1)$-dimensional faces $\tau$ and $\mu$ of $\sigma$ with $\operatorname{dim}(\tau \cap \mu)=r-2$.

Proof. We can define distance $\overline{v w}$ on $S_{C}=\{v \in C \mid v \cdot v=1\} \simeq D_{C}$ by $\cosh \overline{v w}=$ $v \cdot w$ and angle $\angle H_{v}^{C} H_{w}^{C}$ of two hyperplanes $H_{v}^{C}=H_{v} \cap S_{C}$ and $H_{w}^{C}=H_{w} \cap S_{C}$ on $S_{C}$ by $\cos \angle H_{v}^{C} H_{w}^{C}=v \cdot w /(\sqrt{-v \cdot v} \sqrt{-w \cdot w})$ for $v, w \in N_{\mathbf{R}}$ with $v \cdot v<0, w \cdot w<0$. Then we may regard $D_{C}$ as a hyperbolic space and $\left(p_{C}\right)_{\mathbf{R}}(\sigma \backslash\{0\})$ as a Coxeter polyhedron, by the conditions 2, 3 and Proposition 7 . Hence we see by [4, Theorem 7.1.3] that the assertions of the theorem hold.

\section{Structure of exceptional sets.}

We keep the notations and the assumptions in the previous section. Let $\sigma$ be an $r$-dimensional rational polyhedral cone satisfying the conditions of Theorem 8 . Let $W=$ $T_{N} \operatorname{emb}(\Sigma)$ be the toric variety associated to the fan $\Sigma$ in Theorem 8 . For a cone $\tau \neq\{0\}$ in $\Sigma$, we denote by $V(\tau)$ the closure of $\operatorname{orb}(\tau)$ in $W$, which is a compact toric variety (see [5, Corollary 1.7]). Let ord : $T_{N} \rightarrow N_{\mathbf{R}}$ be the homomorphism induced by $-\log ||$ : $\mathbf{C}^{\times} \rightarrow \mathbf{R}$. Let $\widetilde{U}$ be the interior of the closure of $\operatorname{ord}^{-1}(C)$ in $W$ and let $\widetilde{X}=W \backslash T_{N}$. Then $\widetilde{U}$ is an open neighborhood of $\widetilde{X}$. Let $\Gamma_{0}$ be a subgroup of $\Gamma$ with a finite index acting on $D_{C}$ freely. Then $\Gamma_{0}$ acts on $\widetilde{U}$ freely. Let $U=\widetilde{U} / \Gamma_{0}$ and let $X=\widetilde{X} / \Gamma_{0}$. Then the cusp singularity $\operatorname{Cusp}\left(C, \Gamma_{0}\right)$ is obtained by contracting $X$ to a point in $U$ (see $\left.[\mathbf{8}]\right)$.

Let $\lambda$ be a face of $\sigma$ with $1 \leq s:=\operatorname{dim} \lambda \leq r-2$, and let $p_{\lambda}: N \rightarrow N /(\mathbf{R} \lambda \cap N)$ be the natural projection. Let $\mu_{1}, \mu_{2}, \ldots, \mu_{l}$ be the $(r-1)$-dimensional faces of $\sigma$ with $\lambda \prec \mu_{i}$ and let $\Gamma_{\lambda}=\left\langle\gamma_{v\left(\mu_{i}\right)} \mid i=1, \ldots, l\right\rangle$. Then $\Gamma_{\lambda}$ acts on $N /(\mathbf{R} \lambda \cap N)$. Let $\Sigma_{\lambda}=\left\{\left(p_{\lambda}\right)_{\mathbf{R}}(\tau) \mid \tau \in \Sigma, \lambda \prec \tau\right\}$. Then $\Sigma_{\lambda}$ is a $\Gamma_{\lambda}$-invariant fan in $N /(\mathbf{R} \lambda \cap N)$. Moreover, $V(\lambda) \simeq T_{N /(\mathbf{R} \lambda \cap N)} \operatorname{emb}\left(\Sigma_{\lambda}\right)$, by [5, Corollary 1.7]. Hence $V(\lambda)$ is non-singular, if and only if so is $\left(p_{\lambda}\right)_{\mathbf{R}}(\sigma)$. 
Now, assume that $\left(p_{\lambda}\right)_{\mathbf{R}}(\sigma)$ is non-singular, i.e., $\left(p_{\lambda}\right)_{\mathbf{R}}(\sigma)=\mathbf{R}_{\geq 0} w_{1}+\mathbf{R}_{\geq 0} w_{2}+\cdots+$ $\mathbf{R}_{\geq 0} w_{r-s}$ for a basis $\left\{w_{1}, w_{2}, \ldots, w_{r-s}\right\}$ of $N /(\mathbf{R} \lambda \cap N)$. Then there exist elements $u_{1}, u_{2}$, $\ldots, u_{r-s}$ in $N \cap \sigma$ with $w_{i}=p_{\lambda}\left(u_{i}\right)$. Let $\left\{u_{r-s+1}, \ldots, u_{r}\right\}$ be a basis of $\mathbf{R} \lambda \cap N$. Then $\left\{u_{1}, u_{2}, \ldots, u_{r}\right\}$ is a basis of $N$. Moreover, so is $\left\{u_{1}, \ldots, u_{i-1}, \gamma_{v\left(\mu_{i}\right)} u_{i}, u_{i+1}, \ldots, u_{r}\right\}$, because $\gamma_{v\left(\mu_{i}\right)}$ is in $G L(N)$ and $\gamma_{v\left(\mu_{i}\right)} u_{j}=u_{j}$ if $i \neq j$. Hence there exist integers $c_{i, j}$ $(1 \leq i \leq r-s, 1 \leq j \leq r)$ with

$$
u_{i}+\gamma_{v\left(\mu_{i}\right)} u_{i}+c_{i, 1} u_{1}+\cdots+c_{i, i-1} u_{i-1}+c_{i, i+1} u_{i+1}+\cdots+c_{i, r} u_{r}=0 .
$$

Therefore,

$$
w_{i}+\gamma_{v\left(\mu_{i}\right)} w_{i}+c_{i, 1} w_{1}+\cdots+c_{i, i-1} w_{i-1}+c_{i, i+1} w_{i+1}+\cdots+c_{i, r-s} w_{r-s}=0 .
$$

These numbers $c_{i, j}$ determine the structure of $V(\lambda)$. Especially, when $s=r-3$, they are nothing but double $\mathbf{Z}$-weights in $[\mathbf{5}, 1.7]$. We easily see that $c_{i, j} \leq 0$. Moreover, $\left|\gamma_{v\left(\mu_{i}\right)} \gamma_{v\left(\mu_{j}\right)}\right|=+\infty$, if $c_{i, j} \leq-2$ and $c_{j, i} \leq-2, c_{i, j}=-1$ and $c_{j, i} \leq-4$ or $c_{i, j}=0$ and $c_{j, i} \neq 0$. Hence if $v\left(\mu_{i}\right) \cdot v\left(\mu_{j}\right) /\left(\sqrt{-v\left(\mu_{i}\right) \cdot v\left(\mu_{i}\right)} \sqrt{-v\left(\mu_{j}\right) \cdot v\left(\mu_{j}\right)}\right)=0,1 / 2,1 / \sqrt{2}$ or $\sqrt{3} / 2$, then $\left\{c_{i, j}, c_{j, i}\right\}=\{0\},\{-1\},\{-1,-2\}$ or $\{-1,-3\}$, respectively, by Proposition 7 .

We explain some examples of $V(\lambda)$ for the convenience of the next section. First, we consider the case $s=r-2$ and $\left(p_{\lambda}\right)_{\mathbf{R}}(\sigma)$ is non-singular. If $c_{1,2}=c_{2,1}=0$, then $V(\lambda) \simeq \mathbf{P}^{1} \times \mathbf{P}^{1}$. If $c_{1,2}=c_{2,1}=-1$, then $V(\lambda) \simeq S_{6}$. If $c_{1,2}=-1$ and $c_{2,1}=-2$ (resp. -3 ), then $V(\lambda) \simeq S_{8}$ (resp. $S_{12}$ ). Here $S_{i}$ are toric surfaces obtained from Coxeter groups as follows (see $[\mathbf{2}, 5.1]$ for the definition of Coxeter group). For each $i=6,8,12$, let $G_{i}$ be a subgroup of $G L(2, \mathbf{Z})$ generated by two elements $g_{1}$ and $g_{2, i}$ defined by

$$
g_{1}=\left(\begin{array}{rr}
-1 & 0 \\
1 & 1
\end{array}\right), \quad g_{2,6}=\left(\begin{array}{rr}
1 & 1 \\
0 & -1
\end{array}\right), \quad g_{2,8}=\left(\begin{array}{rr}
1 & 2 \\
0 & -1
\end{array}\right), \quad g_{2,12}=\left(\begin{array}{rr}
1 & 3 \\
0 & -1
\end{array}\right)
$$

Then $G_{i}$ are Coxeter groups with $\left|G_{i}\right|=i$. Let $\Lambda_{i}=\left\{\right.$ faces of $\left.g \mathbf{R}_{\geq 0}^{2} \mid g \in G_{i}\right\}$. Then $\Lambda_{i}$ is a non-singular fan for each $i$. Let $S_{i}=T_{\mathbf{Z}^{2}} \operatorname{emb}\left(\Lambda_{i}\right)$ be the compact toric surface associated to the fan $\Lambda_{i}$. Then the complement of the algebraic torus in $S_{6}$, is a cycle of 6 rational curves with the self-intersection numbers all equal to -1 . The complement of the algebraic torus in $S_{8}$ (resp. $S_{12}$ ), is a cycle of 8 (resp. 12) rational curves with the self-intersection numbers repeating $-1,-2$ (resp. $-1,-3$ ).

Next, we consider the case $s=r-3$ and assume that $\left(p_{\lambda}\right)_{\mathbf{R}}(\sigma)$ is non-singular except the case (7). We denote by $V_{i}$ the toric variety $V(\lambda)$ in (i), which appears in the following sections as an irreducible component of the exceptional set of a resolution of 4-dimensional cusp singularities.

(1a) If $c_{1,2}=c_{2,1}=0, c_{1,3}=c_{3,1}=c_{3,2}=-1, c_{2,3}=-2$, then the complement of the algebraic torus in $V_{1 a}$, consists of 26 toric surfaces 6,8 and 12 of which are biholomorphic to $S_{8}, S_{6}$ and $\mathbf{P}^{1} \times \mathbf{P}^{1}$, respectively (see Figure 3 ). The selfintersection numbers $\left(E_{\mid V}\right)^{2}$ in irreducible components $V \simeq S_{8}$ of rational curves $E=V \cdot W$, are equal to -2 and -1 , if $W \simeq \mathbf{P}^{1} \times \mathbf{P}^{1}$ and $S_{6}$, respectively.

(1b) If $c_{1,2}=c_{2,1}=0, c_{1,3}=c_{3,1}=c_{2,3}=-1, c_{3,2}=-2$, then the complement of the algebraic torus in $V_{1 b}$, consists of 26 toric surfaces 6,8 and 12 of which 


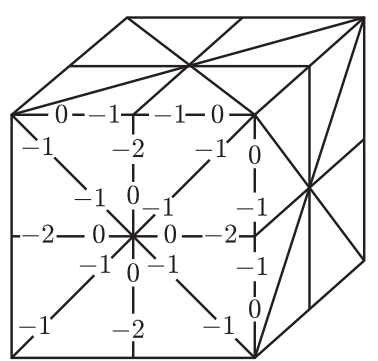

Figure 3.

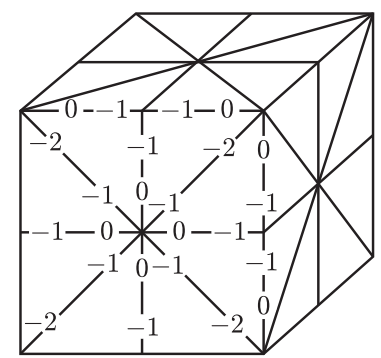

Figure 4.

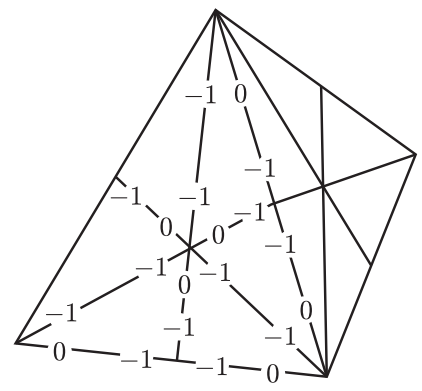

Figure 5.

are biholomorphic to $S_{8}, S_{6}$ and $\mathbf{P}^{1} \times \mathbf{P}^{1}$, respectively (see Figure 4 ). The selfintersection numbers $\left(E_{\mid V}\right)^{2}$ in irreducible components $V \simeq S_{8}$ of rational curves $E=V \cdot W$, are equal to -1 and -2 , if $W \simeq \mathbf{P}^{1} \times \mathbf{P}^{1}$ and $S_{6}$, respectively.

(2) If $c_{1,2}=c_{2,1}=0, c_{1,3}=c_{3,1}=c_{3,2}=c_{2,3}=-1$, then the complement of the algebraic torus in $V_{2}$, consists of 14 toric surfaces 8 and 6 of which are biholomorphic to $S_{6}$ and $\mathbf{P}^{1} \times \mathbf{P}^{1}$, respectively (see Figure 5).

(3) If $c_{1,2}=c_{2,1}=c_{1,3}=c_{3,1}=0, c_{2,3}=c_{3,2}=-1$ then $V_{3} \simeq \mathbf{P}^{1} \times S_{6}$.

(4) If $c_{1,2}=c_{2,1}=c_{1,3}=c_{3,1}=0, c_{2,3}=-1, c_{3,2}=-2$ then $V_{4} \simeq \mathbf{P}^{1} \times S_{8}$.

(5) If $c_{1,2}=c_{2,1}=c_{1,3}=c_{3,1}=0, c_{2,3}=-1, c_{3,2}=-3$ then $V_{5} \simeq \mathbf{P}^{1} \times S_{12}$.

(6) If $c_{i, j}=0$ for all $i, j$, then $V_{6} \simeq \mathbf{P}^{1} \times \mathbf{P}^{1} \times \mathbf{P}^{1}$.

(7) If $\left(p_{\lambda}\right)_{\mathbf{R}}(\sigma)$ is simplicial, $v\left(\mu_{i}\right) \cdot v\left(\mu_{j}\right)=0$ for $1 \leq i<j \leq 3$ and $u_{1}=\mathbf{f}_{1}, u_{2}=$ $\mathbf{f}_{1}+2 \mathbf{f}_{2}, u_{3}=\mathbf{f}_{3}$ for a basis $\left\{\mathbf{f}_{1}, \mathbf{f}_{2}, \ldots, \mathbf{f}_{r}\right\}$ of $N$, then $V_{7} \simeq \mathbf{P}^{1} \times\left(\mathbf{P}^{1} \times \mathbf{P}^{1} /(-1,-1)\right)$.

\section{Examples with quadratic $C$.}

We fix $r=4$, throughout the rest of this paper.

ExAmple 1. Let $P\left(x_{1}, x_{2}, x_{3}, x_{4}\right)=-x_{1}^{2}-x_{2}^{2}-x_{3}^{2}+7 x_{4}^{2}$. Let $\sigma$ be the cone generated by the following six elements in $N$.

$$
u_{1}=\left[\begin{array}{l}
0 \\
0 \\
0 \\
1
\end{array}\right], u_{2}=\left[\begin{array}{l}
7 \\
7 \\
0 \\
4
\end{array}\right], u_{3}=\left[\begin{array}{l}
7 \\
7 \\
7 \\
5
\end{array}\right], u_{4}=\left[\begin{array}{c}
14 \\
7 \\
0 \\
6
\end{array}\right], u_{5}=\left[\begin{array}{c}
21 \\
7 \\
7 \\
9
\end{array}\right], u_{6}=\left[\begin{array}{l}
7 \\
0 \\
0 \\
3
\end{array}\right]
$$

Let $C$ be the connected component of $C_{P}$ containing $u_{1}$. Then $\sigma \backslash\{0\} \subset C$. Let

$$
v_{1}=\left[\begin{array}{c}
-1 \\
1 \\
0 \\
0
\end{array}\right], v_{2}=\left[\begin{array}{c}
0 \\
-1 \\
1 \\
0
\end{array}\right], v_{3}=\left[\begin{array}{c}
0 \\
0 \\
-1 \\
0
\end{array}\right], v_{4}=\left[\begin{array}{l}
3 \\
0 \\
0 \\
1
\end{array}\right], v_{5}=\left[\begin{array}{l}
2 \\
2 \\
1 \\
1
\end{array}\right]
$$




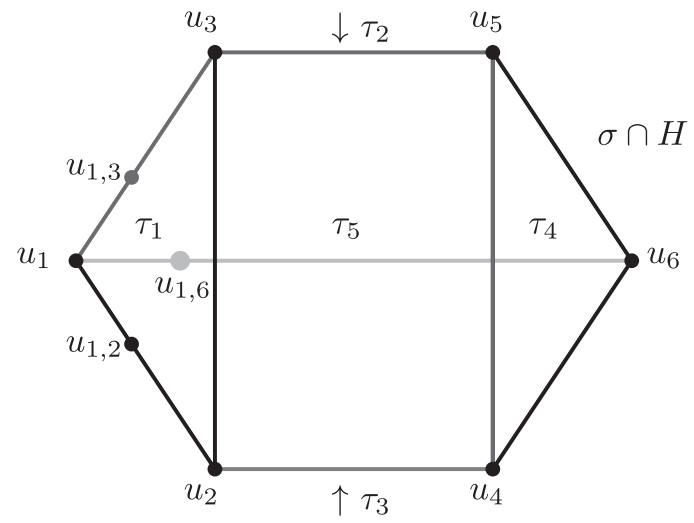

Figure 6 .

Then $\tau_{i}:=\sigma \cap H_{v_{i}}(i=1, \ldots, 5)$ are 3-dimensional faces of $\sigma$ (see Figure 6 which shows the intersection with a hyperplane $H$ ). Moreover, we see by Proposition 5 and easy calculation that $v\left(\tau_{i}\right)=v_{i}$ satisfy the conditions 2,3 of Theorem 8 . Let $\Sigma$ be the fan in Theorem 8 defined for this $\sigma$. Then $V(\lambda)$ are singularities in $T_{N} \operatorname{emb}(\Sigma)$ for all cones $\lambda$ in $\Sigma$ with $\operatorname{dim} \lambda \geq 2$. Noting that $\sigma^{\vee}$ is spanned by $i\left(v_{1}\right), i\left(v_{2}\right), \ldots, i\left(v_{5}\right)$, where $i: N \rightarrow M$ is the homomorphism satisfying $\langle i(x), y\rangle=B_{P}(x, y)$, we see that all 3-dimensional faces of $\sigma^{\vee}$ are non-singular. Let $\lambda=\mathbf{R}_{\geq 0} u_{1}$ and let

$$
u_{1,2}=\left[\begin{array}{l}
1 \\
1 \\
0 \\
1
\end{array}\right], u_{1,3}=\left[\begin{array}{l}
1 \\
1 \\
1 \\
1
\end{array}\right], u_{1,6}=\left[\begin{array}{l}
1 \\
0 \\
0 \\
1
\end{array}\right] \text {. }
$$

Then $\left\{u_{1}, u_{1,2}, u_{1,3}, u_{1,6}\right\}$ is a basis of $N$ and $\left(p_{\lambda}\right)_{\mathbf{R}}(\sigma)=\mathbf{R}_{\geq 0} p_{\lambda}\left(u_{1,2}\right)+\mathbf{R}_{\geq 0} p_{\lambda}\left(u_{1,3}\right)+$ $\mathbf{R}_{\geq 0} p_{\lambda}\left(u_{1,6}\right)$. Moreover, we see by easy calculation that the relations $u_{1,2}+\gamma_{v_{2}} u_{1,2}-$ $u_{1,3}-u_{1,6}=0, u_{1,3}+\gamma_{v_{3}} u_{1,3}-2 u_{1,2}=0$ and $u_{1,6}+\gamma_{v_{1}} u_{1,6}-u_{1}-u_{1,2}=0$ hold. Hence $V(\lambda)$ is biholomorphic to $V_{1 a}$ in the previous section. Since $v_{1} \cdot v_{3}=v_{1} \cdot v_{5}=0$, $v_{3} \cdot v_{5}=1, v_{3} \cdot v_{3}=-1$ and $v_{5} \cdot v_{5}=-2, V\left(\mathbf{R}_{\geq 0} u_{2}\right)$ is biholomorphic to $V_{4}$. We see by similar calculation that $V\left(\mathbf{R}_{\geq 0} u_{i}\right)$ are biholomorphic to $V_{2}, V_{1 a}, V_{2}$ and $V_{4}$ for $i=3,4,5$ and 6 , respectively.

Example 2. Let $P\left(x_{1}, x_{2}, x_{3}, x_{4}\right)=-x_{1}^{2}-x_{2}^{2}-x_{3}^{2}+15 x_{4}^{2}$. Then the cone $\sigma$ defined by $v_{1}, v_{2}, \ldots, v_{6}$, satisfies the conditions of Theorem 8 , where

$$
v_{1}=\left[\begin{array}{c}
-1 \\
1 \\
0 \\
0
\end{array}\right], v_{2}=\left[\begin{array}{c}
0 \\
-1 \\
1 \\
0
\end{array}\right], v_{3}=\left[\begin{array}{c}
0 \\
0 \\
-1 \\
0
\end{array}\right], v_{4}=\left[\begin{array}{l}
5 \\
0 \\
0 \\
1
\end{array}\right], v_{5}=\left[\begin{array}{l}
3 \\
3 \\
0 \\
1
\end{array}\right], v_{6}=\left[\begin{array}{l}
3 \\
2 \\
2 \\
1
\end{array}\right]
$$

(see Figure 7). We can verify that the divisors corresponding to the vertices attached (i) are biholomorphic to $V_{i}$ in the previous section. For example, $v_{2} \cdot v_{4}=v_{2} \cdot v_{6}=v_{4} \cdot v_{6}=0$, 
$\left(\mathbf{R} w_{2}+\mathbf{R} w_{i}\right) \cap M=\mathbf{Z} w_{2}+\mathbf{Z} w_{i}$ for $i=4,6$ and $\left[\left(\mathbf{R} w_{4}+\mathbf{R} w_{6}\right) \cap M: \mathbf{Z} w_{4}+\mathbf{Z} w_{6}\right]=2$, where $w_{i}(i=2,4,6)$ are the elements in $M$ satisfying $\left\langle w_{2}, x\right\rangle=B_{P}\left(v_{2}, x\right),\left\langle w_{4}, x\right\rangle=$ $(1 / 5) B_{P}\left(v_{4}, x\right)$ and $\left\langle w_{6}, x\right\rangle=B_{P}\left(v_{6}, x\right)$. Hence $V\left(\tau_{2} \cap \tau_{4} \cap \tau_{6}\right)$ is biholomorphic to $V_{7}$, where $\tau_{i}=\sigma \cap H_{v_{i}}$.

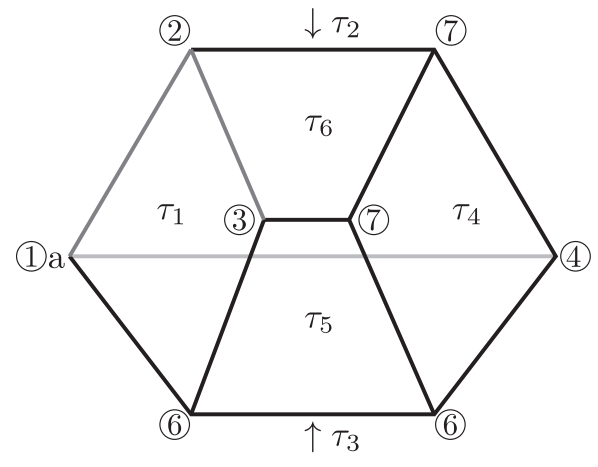

Figure 7.

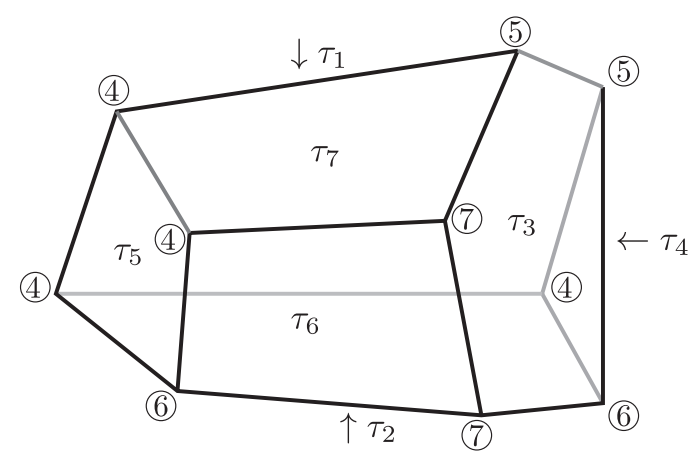

Figure 8 .

ExAmple 3. Let $P\left(x_{1}, x_{2}, x_{3}, x_{4}\right)=-3 x_{1}^{2}-3 x_{2}^{2}-5 x_{3}^{2}+x_{4}^{2}$. Then the cone $\sigma$ defined by $v_{1}, v_{2}, \ldots, v_{6}$, where

$$
\begin{gathered}
v_{1}=\left[\begin{array}{c}
1 \\
-1 \\
0 \\
0
\end{array}\right], \quad v_{2}=\left[\begin{array}{c}
-1 \\
0 \\
0 \\
0
\end{array}\right], \quad v_{3}=\left[\begin{array}{l}
0 \\
1 \\
0 \\
1
\end{array}\right], \quad v_{4}=\left[\begin{array}{c}
0 \\
0 \\
-1 \\
0
\end{array}\right], \\
v_{5}=\left[\begin{array}{l}
0 \\
0 \\
1 \\
2
\end{array}\right], \quad v_{6}=\left[\begin{array}{l}
0 \\
5 \\
6 \\
15
\end{array}\right], \quad v_{7}=\left[\begin{array}{l}
1 \\
1 \\
1 \\
3
\end{array}\right]
\end{gathered}
$$

(see Figure 8).

\section{An example with non-quadratic $C$.}

We fix a basis $\left\{\mathbf{e}_{1}, \mathbf{e}_{2}, \mathbf{e}_{3}, \mathbf{e}_{4}\right\}$ of $N$. Let $\gamma_{i}$ be the elements in $G L(N)$ defined by the following relations for $i=1,2,3,4 . \gamma_{i} \mathbf{e}_{j}=\mathbf{e}_{j}$ if $i \neq j$ and

$\gamma_{1} \mathbf{e}_{1}=-\mathbf{e}_{1}+\mathbf{e}_{2}+2 \mathbf{e}_{3}, \gamma_{2} \mathbf{e}_{2}=\mathbf{e}_{1}-\mathbf{e}_{2}+\mathbf{e}_{4}, \gamma_{3} \mathbf{e}_{3}=\mathbf{e}_{1}-\mathbf{e}_{3}+\mathbf{e}_{4}, \gamma_{4} \mathbf{e}_{4}=2 \mathbf{e}_{2}+\mathbf{e}_{3}-\mathbf{e}_{4}$.

Then $\Gamma_{6}=\left\langle\gamma_{i} \mid i=1,2,3,4\right\rangle$ is a Coxeter group with the relations: $\gamma_{i}^{2}=1$ and

$$
(*)\left(\gamma_{1} \gamma_{2}\right)^{3}=\left(\gamma_{3} \gamma_{4}\right)^{3}=\left(\gamma_{1} \gamma_{3}\right)^{4}=\left(\gamma_{2} \gamma_{4}\right)^{4}=\left(\gamma_{1} \gamma_{4}\right)^{2}=\left(\gamma_{2} \gamma_{3}\right)^{2}=1 .
$$

Hence the Dynkin diagram of $\Gamma_{6}$ is Figure 9 (see $[\mathbf{2}, 2.3]$ for the definition of Dynkin diagram). Let $\sigma=\mathbf{R}_{\geq 0} \mathbf{e}_{1}+\mathbf{R}_{\geq 0} \mathbf{e}_{2}+\mathbf{R}_{\geq 0} \mathbf{e}_{3}+\mathbf{R}_{\geq 0} \mathbf{e}_{4}$ and let $\tau_{i}$ be the 3-dimensional face of $\sigma$ which does not contain $\mathbf{e}_{i}$ for each $i$. Then $\gamma_{i}$ is a reflection with respect to 
the hyperplane containing $\tau_{i}$. Moreover, the entries $a_{i j}$ of the Cartan matrix in [10], are equal to $-c_{j i}$ if $i \neq j$, where $c_{j i}$ are the coefficients in the above relations $\gamma_{j} \mathbf{e}_{j}=\sum c_{j i} \mathbf{e}_{i}$, because $2 \mathbf{e}_{j}-\sum_{i \neq j} c_{j i} \mathbf{e}_{i}$ is an eigenvector of $\gamma_{j}$ with the eigenvalue -1 . Hence $a_{14}=$ $a_{41}=a_{23}=a_{32}=0, a_{12} \cdot a_{21}=a_{34} \cdot a_{43}=1, a_{13} \cdot a_{31}=a_{24} \cdot a_{42}=2$. Therefore, $C_{6}=$ $\bigcup_{\gamma \in \Gamma_{6}} \gamma \sigma \backslash\{0\}$ is an open strongly convex cone in $N_{\mathbf{R}}$ and $\Sigma_{6}=\left\{\gamma \tau \mid \gamma \in \Gamma_{6}, \tau \prec \sigma\right\}$ is a $\Gamma_{6}$-invariant fan with $\left|\Sigma_{6}\right|=C_{6} \cup\{0\}$, by [10, Theorem 1]. Moreover, $C_{6}$ is not quadratic, by $\left[\mathbf{1 0}\right.$, Theorem 6]. Since $\sigma$ is non-singular, so is $T_{N} \operatorname{emb}\left(\Sigma_{6}\right)$. The 3-dimensional toric variety $V\left(\mathbf{R}_{\geq 0} \mathbf{e}_{i}\right)$ is biholomorphic to $V_{1 a}$ (resp. $V_{1 b}$ ) in Section 4 for $i=2,3$ (resp. 1,4). The intersection $V\left(\mathbf{R}_{\geq 0} \mathbf{e}_{i}\right) \cap V\left(\mathbf{R}_{\geq 0} \mathbf{e}_{j}\right)=V\left(\mathbf{R}_{\geq 0} \mathbf{e}_{i}+\mathbf{R}_{\geq 0} \mathbf{e}_{j}\right)$ is the toric surface corresponding to the Coxeter group generated by $\left\{\gamma_{k}, \gamma_{l}\right\}$ for $\{k, l\}=\{1,2,3,4\} \backslash\{i, j\}$. Hence it is biholomorphic to $\mathbf{P}^{1} \times \mathbf{P}^{1}$ if $(i, j)=(2,3),(1,4), S_{6}$ if $(i, j)=(3,4),(1,2)$ and $S_{8}$ if $(i, j)=(2,4),(1,3)$ by $(*)$. Note that $V\left(\mathbf{R}_{\geq 0} \mathbf{e}_{i}\right) \cap V\left(\mathbf{R}_{\geq 0} \mathbf{e}_{j}\right)$ is biholomorphic to $\mathbf{P}^{1} \times \mathbf{P}^{1}$, if and only if $V\left(\mathbf{R}_{\geq 0} \mathbf{e}_{i}\right)$ and $V\left(\mathbf{R}_{\geq 0} \mathbf{e}_{j}\right)$ are biholomorphic.

REMARK. Let $\Gamma_{6}^{\prime}, \Sigma_{6}^{\prime}$ and $C_{6}^{\prime}$ be the subgroup of $G L(N)$, the fan and the cone in $N_{\mathbf{R}}$, respectively, obtained by transposing the coefficients $c_{2,4}=1$ and $c_{4,2}=2$ in the above relations $\gamma_{i} \mathbf{e}_{i}=\sum c_{i j} \mathbf{e}_{j}$. Then the irreducible components of $T_{N} \operatorname{emb}\left(\Sigma_{6}^{\prime}\right) \backslash T_{N}$ are isomorphic to those of $T_{N} \operatorname{emb}\left(\Sigma_{6}\right) \backslash T_{N}$. However, they intersect to each other in a different way. $V\left(\mathbf{R}_{\geq 0} \mathbf{e}_{i}\right)$ are biholomorphic to $V_{1 a}$ (resp. $V_{1 b}$ ) for $i=1,2$ (resp. $3,4)$. Hence $V\left(\mathbf{R}_{\geq 0} \mathbf{e}_{i}\right) \cap V\left(\mathbf{R}_{\geq 0} \mathbf{e}_{j}\right)$ is biholomorphic to $S_{6}$, if and only if $V\left(\mathbf{R}_{\geq 0} \mathbf{e}_{i}\right)$ and $V\left(\mathbf{R}_{\geq 0} \mathbf{e}_{j}\right)$ are biholomorphic. However, the following consideration for $\left(C_{6}, \Gamma_{6}\right)$ holds also for $\left(C_{6}^{\prime}, \Gamma_{6}^{\prime}\right)$, because the relations in $(*)$ do not change.

Hereafter, we simply write $\Gamma, \Sigma$ and $C$ for $\Gamma_{6}, \Sigma_{6}$ and $C_{6}$, respectively.

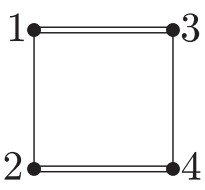

Figure 9

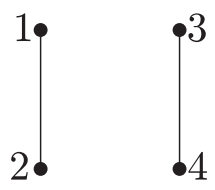

Figure 10.

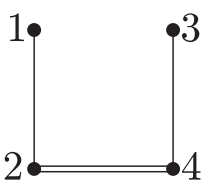

Figure 11.

TheORem 9. There exists a subgroup $\Gamma^{0}$ of $\Gamma$ of index 48 which acts on $D_{C}$ freely. Conversely, if a subgroup $\Gamma^{\prime}$ of $\Gamma$ acts on $D_{C}$ freely, then $\Gamma^{\prime}$ is of index at least 48.

Let $\Gamma^{i}=\left\langle\gamma_{j} \mid 1 \leq j \leq 4, j \neq i\right\rangle$ for each $i$. Then $\Gamma^{i}$ is the stabilizer of $\mathbf{R}_{\geq 0} \mathbf{e}_{i}$ and $\left|\Gamma^{i}\right|=48$. Hence the second assertion in the above theorem holds. Let $\Delta=$ $\left\{p_{C}(\tau \backslash\{0\}) \mid \tau \in \Sigma, \tau \neq\{0\}\right\}$. Then $\Delta$ is a $\Gamma$-invariant tetrahedral decomposition of $D_{C}$. If we get $\Gamma^{0}$ in the above theorem, then $\Delta / \Gamma^{0}$ is a tetrahedral decomposition of the 3-dimensional compact topological manifold $D_{C} / \Gamma^{0}$ consisting of 48 tetrahedra. Since $\Delta / \Gamma^{0}$ has $48 \cdot 4 /\left|\Gamma^{i}\right|=4$ vertices, there exists a resolution of the cusp singularity $\operatorname{Cusp}\left(C, \Gamma^{0}\right)$ with an exceptional set consisting of 4 irreducible components. The rest of this section is devoted to the proof of the first assertion in the above theorem.

Let $\gamma_{i}^{\prime}$ be the elements in $G L(N)$ defined by the following relations for $i=1,2,3,4$. $\gamma_{i}^{\prime} \mathbf{e}_{j}=\mathbf{e}_{j}$ if $i \neq j$ and 


$$
\gamma_{1}^{\prime} \mathbf{e}_{1}=-\mathbf{e}_{1}+\mathbf{e}_{2}, \gamma_{2}^{\prime} \mathbf{e}_{2}=\mathbf{e}_{1}-\mathbf{e}_{2}, \gamma_{3}^{\prime} \mathbf{e}_{3}=-\mathbf{e}_{3}+\mathbf{e}_{4}, \gamma_{4}^{\prime} \mathbf{e}_{4}=\mathbf{e}_{3}-\mathbf{e}_{4}
$$

Then $\Gamma^{\prime}=\left\langle\gamma_{i}^{\prime} \mid i=1,2,3,4\right\rangle$ is a Coxeter group with the relations: $\gamma_{i}^{\prime 2}=1$ and

$$
\left(\gamma_{1}^{\prime} \gamma_{2}^{\prime}\right)^{3}=\left(\gamma_{3}^{\prime} \gamma_{4}^{\prime}\right)^{3}=\left(\gamma_{1}^{\prime} \gamma_{3}^{\prime}\right)^{2}=\left(\gamma_{2}^{\prime} \gamma_{4}^{\prime}\right)^{2}=\left(\gamma_{1}^{\prime} \gamma_{4}^{\prime}\right)^{2}=\left(\gamma_{2}^{\prime} \gamma_{3}^{\prime}\right)^{2}=1 .
$$

Hence the Dynkin diagram of $\Gamma^{\prime}$ is Figure 10, $\Gamma^{\prime} \simeq D_{3} \times D_{3}$ and there exists a surjective homomorphism $q: \Gamma \rightarrow \Gamma^{\prime}$ sending $\gamma_{i}$ to $\gamma_{i}^{\prime}$. Let $\Delta^{\prime}=\left\{p\left(\gamma^{\prime} \tau \backslash\{0\}\right) \mid \gamma^{\prime} \in \Gamma^{\prime}, \tau \prec\right.$ $\sigma, \tau \neq\{0\}\}$, where $p: N_{\mathbf{R}} \backslash\{0\} \rightarrow S^{3}$ is the natural projection. Then $\Delta^{\prime}$ is a tetrahedral decomposition of $S^{3}$ with 36 tetrahedra. Let $\tilde{f}: C \cup\{0\} \rightarrow N_{\mathbf{R}}$ be the piecewise linear map defined by $\tilde{f}(x)=q(\gamma) \gamma^{-1} x$, if $x$ is in $\gamma \sigma$ for an element $\gamma$ in $\Gamma$. Then $\tilde{f}$ induces a Galois covering $f: D_{C} \rightarrow S^{3}$ with $f(\gamma x)=q(\gamma) f(x)$ for any element $\gamma$ in $\Gamma$, ramifying only along $\Xi_{13} \cup \Xi_{24}$, where $\Xi_{i j}=\bigcup_{\gamma^{\prime} \in \Gamma^{\prime}} p\left(\gamma^{\prime}\left(\mathbf{R}_{\geq 0} \mathbf{e}_{i}+\mathbf{R}_{\geq 0} \mathbf{e}_{j}\right) \backslash\{0\}\right)$, because $\left\langle\gamma_{i}, \gamma_{j}\right\rangle$ are the stabilizers of $\mathbf{R}_{\geq 0} \mathbf{e}_{k}+\mathbf{R}_{\geq 0} \mathbf{e}_{l}$, where $\{k, l\}=\{1,2,3,4\} \backslash\{i, j\}$, $q\left(\left(\gamma_{2} \gamma_{4}\right)^{2}\right)=q\left(\left(\gamma_{1} \gamma_{3}\right)^{2}\right)=1$ and the restriction of $q$ to $\left\langle\gamma_{i}, \gamma_{j}\right\rangle$ is an isomorphism if $(i, j) \neq(1,3),(2,4)$. Moreover, $\Delta$ is the pull-back of $\Delta^{\prime}$ under $f$.
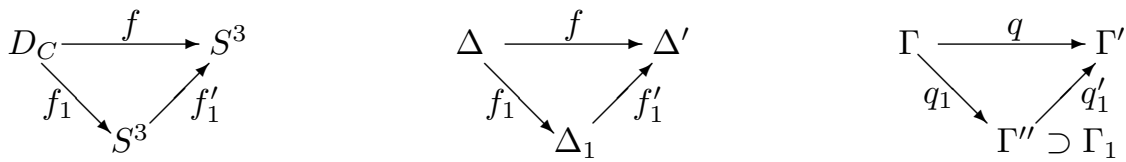

Let $\Gamma^{\prime \prime}=\left\langle\gamma_{i}^{\prime \prime} \mid i=1,2,3,4\right\rangle$, where $\gamma_{1}^{\prime \prime}=\gamma_{1}^{\prime}, \gamma_{2}^{\prime \prime}=\gamma_{2}, \gamma_{3}^{\prime \prime}=\gamma_{3}^{\prime}, \gamma_{4}^{\prime \prime}=\gamma_{4}$. Then $\Gamma^{\prime \prime}$ is a Coxeter group whose Dynkin diagram is Figure 11 and there exist surjective homomorphisms $q_{1}: \Gamma \rightarrow \Gamma^{\prime \prime}$ sending $\gamma_{i}$ to $\gamma_{i}^{\prime \prime}$ and $q_{1}^{\prime}: \Gamma^{\prime \prime} \rightarrow \Gamma^{\prime}$ sending $\gamma_{i}^{\prime \prime}$ to $\gamma_{i}^{\prime}$ with $q=q_{1}^{\prime} \circ q_{1}$. We can define Galois coverings $f_{1}: D_{C} \rightarrow S^{3}$ and $f_{1}^{\prime}: S^{3} \rightarrow S^{3}$ such that $f_{1}^{\prime}\left(\gamma^{\prime \prime} x\right)=q_{1}^{\prime}\left(\gamma^{\prime \prime}\right) f_{1}^{\prime}(x)$ for any element $\gamma^{\prime \prime}$ in $\Gamma^{\prime \prime}$ and that $f_{1}^{\prime} \circ f_{1}=f$, in a similar way as $f$. Then $f_{1}^{\prime}$ ramifies only along $\Xi_{13}, \operatorname{Gal}\left(f_{1}^{\prime}\right)=\operatorname{ker}\left(q_{1}^{\prime}\right)$ and $\Delta_{1}=\left\{p\left(\gamma^{\prime \prime} \tau \backslash\{0\}\right) \mid \gamma^{\prime \prime} \in\right.$ $\left.\Gamma^{\prime \prime}, \tau \prec \sigma, \tau \neq\{0\}\right\}$ is the pull-back of $\Delta^{\prime}$ under $f_{1}^{\prime}$. Let $\gamma_{0}^{\prime \prime}=\gamma_{1}^{\prime \prime} \gamma_{2}^{\prime \prime} \gamma_{3}^{\prime \prime} \gamma_{4}^{\prime \prime}$.

LEMMA. There exists a normal subgroup $\Gamma_{1}$ of $\operatorname{ker}\left(q_{1}^{\prime}\right)$ acting on $S^{3}$ freely with $\operatorname{ker}\left(q_{1}^{\prime}\right) / \Gamma_{1} \simeq \mathbf{Z}_{2} \oplus \mathbf{Z}_{2}, \gamma_{0}^{\prime \prime 3} \in \Gamma_{1}$ and $\gamma_{0}^{\prime \prime} \Gamma_{1} \gamma_{0}^{\prime \prime-1}=\Gamma_{1}$.

Proof. $\quad$ Let $\mathbb{P}$ be the convex hull of the 24 points

$$
\left(\begin{array}{c} 
\pm 2 \\
0 \\
0 \\
0
\end{array}\right), \quad\left(\begin{array}{c}
0 \\
\pm 2 \\
0 \\
0
\end{array}\right), \quad\left(\begin{array}{c}
0 \\
0 \\
\pm 2 \\
0
\end{array}\right), \quad\left(\begin{array}{c}
0 \\
0 \\
0 \\
\pm 2
\end{array}\right), \quad\left(\begin{array}{l} 
\pm 1 \\
\pm 1 \\
\pm 1 \\
\pm 1
\end{array}\right)
$$

in $\mathbf{R}^{4}$. Then the boundary $\partial \mathbb{P}$ of $\mathbb{P}$ consists of 24 octahedra which are on the hyperplanes defined by $\pm x_{i} \pm x_{j}=2(1 \leq i<j \leq 4)$, and is a regular polyhedron of type $(3,4,3)$ (see $[\mathbf{1}, 8.2])$. For example, an octahedron has 6 vertices ${ }^{t}(2,0,0,0),{ }^{t}(0,2,0,0),{ }^{t}(1,1, \pm 1, \pm 1)$. Let $\square$ be the barycentric subdivision of the octahedral decomposition $p(\partial \mathbb{P})$ of $S^{3}$ which is the image of $\partial \mathbb{P}$ under the projection $p: \mathbf{R}^{4} \backslash\{0\} \rightarrow S^{3}$. Let $h: S^{3} \rightarrow S^{3}$ be the homeomorphism induced by the linear transformation $\tilde{h}$ sending $\mathbf{e}_{1}, \mathbf{e}_{2}, \mathbf{e}_{3}$ and $\mathbf{e}_{4}$ to ${ }^{t}(1,1,0,0),{ }^{t}(2,1,1,0),{ }^{t}(1,1,1,1)$ and ${ }^{t}(2,2,2,0)$, respectively. Then $h \Delta_{1}$ coincides 

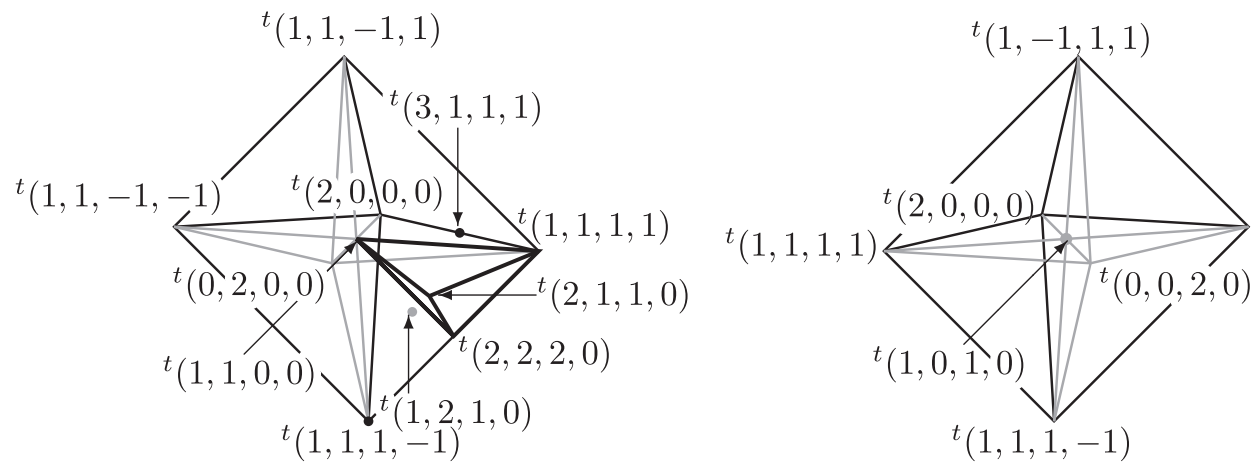

Figure 12 .

with $\square$, because $\tilde{h}\left(\gamma_{1}^{\prime \prime} \mathbf{e}_{1}\right)={ }^{t}(1,0,1,0), \tilde{h}\left(\gamma_{2}^{\prime \prime} \mathbf{e}_{2}\right)={ }^{t}(1,2,1,0), \tilde{h}\left(\gamma_{3}^{\prime \prime} \mathbf{e}_{3}\right)={ }^{t}(1,1,1,-1)$ and $\tilde{h}\left(\gamma_{4}^{\prime \prime} \mathbf{e}_{4}\right)={ }^{t}(3,1,1,1)$ (see Figure 12). Moreover, $h\left(f_{1}^{\prime-1}\left(\Xi_{13}\right)\right)$ is the union of the diagonals of the octahedra on $p(\partial \mathbb{P})$. Since the barycentric subdivision of an octahedron has 48 tetrahedra, $\left|\Gamma^{\prime \prime}\right|=24 \cdot 48=1152$. Since $\operatorname{ker}\left(q_{1}^{\prime}\right)$ is generated by the conjugates of $\left(\gamma_{2}^{\prime \prime} \gamma_{4}^{\prime \prime}\right)^{2}$, whose fixed points are contained in $f_{1}^{\prime-1}\left(\Xi_{13}\right)$ and $\left|\operatorname{ker}\left(q_{1}^{\prime}\right)\right|=\left|\Gamma^{\prime \prime}\right| /\left|\Gamma^{\prime}\right|=$ $1152 / 36=32, \tilde{h} \operatorname{ker}\left(q_{1}^{\prime}\right) \tilde{h}^{-1}$ consists of the following 32 matrices, where $\epsilon_{i}= \pm 1$ and $\epsilon_{1} \epsilon_{2} \epsilon_{3} \epsilon_{4}=1$.

$$
\left(\begin{array}{cccc}
\epsilon_{1} & 0 & 0 & 0 \\
0 & \epsilon_{2} & 0 & 0 \\
0 & 0 & \epsilon_{3} & 0 \\
0 & 0 & 0 & \epsilon_{4}
\end{array}\right),\left(\begin{array}{cccc}
0 & \epsilon_{1} & 0 & 0 \\
\epsilon_{2} & 0 & 0 & 0 \\
0 & 0 & 0 & \epsilon_{3} \\
0 & 0 & \epsilon_{4} & 0
\end{array}\right), \quad\left(\begin{array}{cccc}
0 & 0 & \epsilon_{1} & 0 \\
0 & 0 & 0 & \epsilon_{2} \\
\epsilon_{3} & 0 & 0 & 0 \\
0 & \epsilon_{4} & 0 & 0
\end{array}\right),\left(\begin{array}{cccc}
0 & 0 & 0 & \epsilon_{1} \\
0 & 0 & \epsilon_{2} & 0 \\
0 & \epsilon_{3} & 0 & 0 \\
\epsilon_{4} & 0 & 0 & 0
\end{array}\right) .
$$

Note that the fixed points of all matrices of order 2 in the above except $-I_{4}$, are contained in the diagonals of the octahedra and that any one of order 4 in the above is the product of two of order 2. The set consisting of $\pm I_{4}, \pm A, \pm B$ and $\pm C$ is a normal subgroup of $\tilde{h} \operatorname{ker}\left(q_{1}^{\prime}\right) \tilde{h}^{-1}$ acting on $S^{3}$ freely, where

$$
A=\left(\begin{array}{cccc}
0 & 1 & 0 & 0 \\
-1 & 0 & 0 & 0 \\
0 & 0 & 0 & 1 \\
0 & 0 & -1 & 0
\end{array}\right), \quad B=\left(\begin{array}{cccc}
0 & 0 & 1 & 0 \\
0 & 0 & 0 & -1 \\
-1 & 0 & 0 & 0 \\
0 & 1 & 0 & 0
\end{array}\right), \quad C=\left(\begin{array}{cccc}
0 & 0 & 0 & 1 \\
0 & 0 & 1 & 0 \\
0 & -1 & 0 & 0 \\
-1 & 0 & 0 & 0
\end{array}\right)
$$

Let $J=\tilde{h} \gamma_{0}^{\prime \prime} \tilde{h}^{-1}$. Then

$$
J=\frac{1}{2}\left(\begin{array}{cccc}
1 & 1 & -1 & 1 \\
1 & -1 & 1 & 1 \\
1 & 1 & 1 & -1 \\
1 & -1 & -1 & -1
\end{array}\right)
$$

Hence $J^{3}=-B, J A J^{-1}=-A, J B J^{-1}=B$ and $J C J^{-1}=-C$. Since $\left|\operatorname{ker}\left(q_{1}^{\prime}\right) / \Gamma_{1}\right|=4$ and $X^{2}=-I_{4}$ for any element $X$ of order 4 in $\tilde{h} \operatorname{ker}\left(q_{1}^{\prime}\right) \tilde{h}^{-1}, \operatorname{ker}\left(q_{1}^{\prime}\right) / \Gamma_{1} \simeq \mathbf{Z}_{2} \oplus \mathbf{Z}_{2}$. 
Let $T_{1}=S^{3} / \Gamma_{1}$ and let $g_{1}^{\prime}: T_{1} \rightarrow S^{3}$ be the Galois covering induced by $f_{1}^{\prime}$. Then $g_{1}^{\prime}$ ramifies only along $\Xi_{13}$. Let $h_{1}: D_{C} \rightarrow T_{1}$ be the composite of $f_{1}$ and the quotient map $S^{3} \rightarrow T_{1}$ under $\Gamma_{1}$. Then $h_{1}$ ramifies only along $g_{1}^{\prime-1}\left(\Xi_{24}\right)$ and $f=g_{1}^{\prime} \circ h_{1}$. Moreover, $\gamma_{0}^{\prime \prime}$ induces an automorphism $\delta_{1}$ on $T_{1}$ with $\left|\delta_{1}\right|=3$, by the above lemma. Let $\gamma_{0}^{\prime}=\gamma_{1}^{\prime} \gamma_{2}^{\prime} \gamma_{3}^{\prime} \gamma_{4}^{\prime}$. Then $\gamma_{0}^{\prime}$ has no fixed points on $S^{3}$ and $q_{1}^{\prime}\left(\gamma_{0}^{\prime \prime}\right)=\gamma_{0}^{\prime}$. Hence $g_{1}^{\prime} \circ \delta_{1}=\gamma_{0}^{\prime} \circ g_{1}^{\prime}$. In a similar way, we obtain Galois coverings $g_{2}^{\prime}: T_{2} \rightarrow S^{3}$ ramifying only along $\Xi_{24}, h_{2}: D_{C} \rightarrow T_{2}$ ramifying only along $g_{2}^{\prime-1}\left(\Xi_{13}\right)$ with $f=g_{2}^{\prime} \circ h_{2}$ and an automorphism $\delta_{2}$ on $T_{2}$ with $\left|\delta_{2}\right|=3$ such that $g_{2}^{\prime} \circ \delta_{2}=\gamma_{0}^{\prime} \circ g_{2}^{\prime}$.

$$
D_{C} \rightarrow T=T_{1} \times_{S^{3}} T_{2} \rightarrow T_{0}=T / G_{0} \rightarrow T_{0} /\left\langle\delta_{0}\right\rangle=D_{C} / \Gamma^{0} \rightarrow S^{3}
$$

Now, to show the existence of a subgroup $\Gamma^{0}$ in the theorem, we construct covering maps as above, where the left three arrows do not ramify and the right one ramifies along $\Xi_{13} \cup \Xi_{24}$. Let $T=T_{1} \times_{S^{3}} T_{2}$ be the fiber product of $g_{1}^{\prime}$ and $g_{2}^{\prime}$. Then $T$ is a topological manifold, because $\Xi_{13} \cap \Xi_{24}=\emptyset$. Since $\operatorname{Gal}\left(g_{i}^{\prime}\right) \simeq \mathbf{Z}_{2} \oplus \mathbf{Z}_{2}$, any bijection between $\operatorname{Gal}\left(g_{1}^{\prime}\right) \backslash\{1\}$ and $\operatorname{Gal}\left(g_{2}^{\prime}\right) \backslash\{1\}$ induces an isomorphism. Hence there exists an isomorphism $\phi: \operatorname{Gal}\left(g_{1}^{\prime}\right) \simeq \operatorname{Gal}\left(g_{2}^{\prime}\right)$ such that $\phi\left(\delta_{1} \gamma \delta_{1}^{-1}\right)=\delta_{2} \phi(\gamma) \delta_{2}^{-1}$ for any element $\gamma$ in $\operatorname{Gal}\left(g_{1}^{\prime}\right)$. Let $G_{0}=\left\{(\gamma, \phi(\gamma)) \mid \gamma \in \operatorname{Gal}\left(g_{1}^{\prime}\right)\right\}$. Then $G_{0}$ has no fixed points on $T$, because $\Xi_{13} \cap \Xi_{24}=\emptyset$. Let $T_{0}=T / G_{0}$ and let $g_{0}^{\prime}: T_{0} \rightarrow S^{3}$ be the covering induced by the natural projection $T \rightarrow S^{3}$. Then $\operatorname{deg} g_{0}^{\prime}=4$, because $\operatorname{deg} g_{i}^{\prime}=4$. Hence the pull-back of $\Delta^{\prime}$ under $g_{0}^{\prime}$, consists of $36 \cdot 4=144$ tetrahedra. Let $h: D_{C} \rightarrow T_{0}$ be the composite of the map $\left(h_{1}, h_{2}\right)$ and the quotient map $T \rightarrow T_{0}$. Then $h$ is a surjective unramified covering, because it does not ramify along $g_{0}^{\prime-1}\left(\Xi_{13} \cup \Xi_{24}\right)$ and $T_{0}$ is a topological manifold. Since $\left(\delta_{1}, \delta_{2}\right) G_{0}\left(\delta_{1}, \delta_{2}\right)^{-1}=G_{0},\left(\delta_{1}, \delta_{2}\right)$ induces an automorphism $\delta_{0}$ on $T_{0}$ with $g_{0}^{\prime} \circ \delta_{0}=\gamma_{0}^{\prime} \circ g_{0}^{\prime}$. Since $\gamma_{0}^{\prime}$ has no fixed points on $S^{3}$, so does $\delta_{0}$ on $T_{0}$. Hence the composite of $h$ and the quotient map $T_{0} \rightarrow T_{0} /\left\langle\delta_{0}\right\rangle$, is the quotient map under a subgroup of $\Gamma$ with the index $144 / 3=48$ acting on $D_{C}$ freely.

\section{References}

[1] H. S. M. Coxeter, Regular Polytopes, 3rd edn., Dover, New York, 1973.

[2] J. E. Humphreys, Reflection groups and Coxeter groups, Cambridge Studies in Advanced Mathematics, 29, Cambridge.

[ 3 ] M. Ishida, Cusp singularities given by reflections of stellable cones, Internat. J. Math., 2 (1991), 635-657.

[4] J. G. Ratcliffe, Foundations of Hyperbolic Manifolds, 2nd edn., GTM, 149, Springer.

[5] T. Oda, Convex Bodies and Algebraic Geometry, Ergebnisse der Mathematik und ihrer Grenzgebiete 3, Folge-Band 15, Springer-Verlag, 1987.

[6] I. Satake, On numerical invariants of arithmetic varieties of Q-rank one, in Automorphic forms of several variables, Taniguchi Symposium, Katata, 1983 (I. Satake and Y. Morita, eds.), Progr. Math., 46, Birkhauser, Basel-Boston-Stuttgart, 1984, 353-369.

[ 7 ] I. Satake and S. Ogata, Zeta functions associated to cones and their special values, Automorphic Forms and Geometry of Arithmetic Varieties, Adv. Stud. Pure Math., 15, 1989, 1-27.

[ 8 ] H. Tsuchihashi, Higher dimensional analogues of periodic continued fractions and cusp singularities, Tohoku Math. J., 35 (1983), 607-639.

[ 9 ] H. Tsuchihashi, Three-Dimensional Cusp Singularities, Complex Analytic Singularities, Adv. Stud. Pure Math., 8, 1986, 649-670.

[10] È. B. Vinberg, Discrete linear groups generated by reflections, Math. USSR Izvestija, 5 (1971), 1083-1119. 
Hiroyasu TsuchiHASHI

Miyagi University of Education 149 Aramaki Aza Aoba Aobaku, Sendai

Miyagi 980-0845, Japan 